\title{
LA DIASPORA LIBANAISE UNE ORGANISATION COMMUNAUTAIRE
}

\begin{abstract}
RÉSUMÉ.- La diaspora libanaise est l'une des grandes diasporas mondiales par son ancienneté et son ampleur. Elle a su transposer des modèles ethniques hors du territoire d'origine, préservant son identité ethnico-religieuse et la solidarité communautaire. La capacité de reconversion de la diaspora libanaise, liée à la structure en réseaux, peut expliquer sa possibilité d'adaptation dans des pays très différents, notamment à partir des activités marchandes et financières. Elle est extrêmement attentive à la situation du Liban lui-même.
\end{abstract}

Amir Abdulkarim

\section{COMMERCE, DIASPORA, ESPACE, IDENTITÉ, LIBAN, RÉSEAU, TERRITOIRE}

\begin{abstract}
The Lebanese diaspora: a community organization.
The Lebanese diaspora today ranks among the first owing to its size and ageold tradition. Ethnic models have been successfully transferred from the homeland while preserving the ethnic and religious identity, as well as the traditions of solidarity, of the community. The adaptability of the Lebanese diaspora, which consists of a network, is perhaps the reason for its capacity for integration in a wide variety of countries, notably in the trade and financial sectors. Great attention is paid within the diaspora to events in Lebanon.
\end{abstract}

DIASPORA, IDENTITY, LEBANON, NETWORK, SPACE, TERRITORY, TRADE

\section{Introduction}

Le terme de diaspora ne souffre pas d'ambiguïté lorsqu'on l'emploie à propos des Libanais installés à l'étranger. Plusieurs caractéristiques, toutes définitions confondues, permettent de placer la diaspora libanaise au rang des diasporas juive, grecque et arménienne. Des exodes massifs ont donné naissance à cette diaspora, aussi peuplée, ou plus, que le Liban même. Elle entretient avec le territoire d'origine des rapports étroits qui contribuent à son identité. Le sentiment d'appartenir à une entité territoriale et à un ensemble identitaire engendre une mémoire et une conscience collective visant à sauvegarder cette identité qui, quelque soit par

\footnotetext{
* Migrinter, URA 1145 CNRS, Université de Poitiers
} 
ailleurs le degré d'intégration, repose sur une volonté de durer en tant que groupe ethnique transmettant un héritage.

Le fonctionnement de la diaspora libanaise dépend de réseaux de relations avec le pays d'origine, outre ceux qui existent entre les différents pôles de la diaspora. Ils sont capables, à n'importe quel moment, de mobiliser les ressources ethniques, matérielles et morales, au service de la communauté. Cet article essaie d'analyser l'interaction de différentes variables telles qu'émigration, espace, temps, identité, dans la constitution de la diaspora libanaise. L'une des questions majeures consiste à savoir dans quelle mesure la diaspora peut représenter une nouvelle forme de restructuration d'une partie de la société d'origine à l'extérieur du territoire national.

\section{La diaspora : l'autre Liban}

La diaspora libanaise compte actuellement 3 à 4 millions de personnes, dispersées dans plus de 70 pays : c'est considérable par rapport à la population restée au pays, un peu plus de 3 millions d'habitants (Hourani, Shehadi, 1992). Cette diaspora résulte des bouleversements historiques qui ont secoué le Liban et le Moyen-Orient, brisant la stabilité des peuplements et des territoires. En 1840 et 1860, des affrontements entre communautés religieuses, derrière lesquels se dessinent des rivalités entre grandes puissances du Moyen-Orient, se sont traduits au Liban par la déstabilisation des sociétés rurales et leur appauvrissement, entretenant un vaste mouvement d'émigration qui s'est amplifié en raison d'une série de crises politiques, sociales el économiques. Entre 1860 et le début du premier conflit mondial en 1914, on estime le nombre d'émigrés libanais à 310000 (Safa, 1960). Le mouvement d'émigration a continué, mais à un moindre rythme, durant et après la guerre. Vers 1930, la diaspora compte près d'un million de Libanais, dans leur grande majorité chrétiens. Près de la moitié se trouvent en Amérique latine, au Brésil et en Argentine surtout ; un quart sont aux États-Unis ; le reste se distribue entre l'Australie et l'Afrique noire. Enfin, à partir des années 1950 et 1960, une " fuite des cerveaux » draine des travailleurs spécialisés, des techniciens et des cadres compétents, non seulement vers les foyers traditionnels de la diaspora, mais aussi vers de nouveaux espaces, notamment les pays du Golfe. Le développement rapide des économies pétrolières y suscite une nouvelle immigration des Libanais, fortement intensifiée par la suite en raison de la guerre civile de 1975 et de ses tragiques conséquences.

La première diaspora libanaise est composée de paysans et de petits propriétaires terriens qui n'arrivent pas à surmonter la crise. Elle compte une forte majorité de maronites, qui ont fui les persécutions et les massacres de 1840 et 1860 . La vague qui a rejoint la diaspora entre les deux guerres mondiales est composée différemment: elle comprend de plus en plus de citadins ou de néocitadins, pour la plupart instruits.

\section{Guerre civile et réémergence de la diaspora}

Le déclenchement de la guerre civile en 1975 a brusquement fortifié la diaspora libanaise, puisque près d'un million de Libanais auraient quitté le pays tout au long des affrontements (1975-1990). D'abord émigration provisoire vers les pays voisins, elle s'est peu à peu transformée en une émigration définitive à destination de pays lointains : la France, les ÉtatsUnis, l'Australie, certains pays d'Afrique occidentale. La prolongation de la guerre, la persistance d'une situation dramatique et d'un climat d'insécurité, notamment à la suite de l'invasion israélienne de 1982, ont accéléré l'exode et changé sa signification en altérant les 
perspectives de retour et en provoquant une «rediasporisation » (Corm, 1989). Les premières années de la guerre ont vu l'émigration des couches supérieures de la société libanaise, mais la persistance de la crise a élargi le mouvement à l'ensemble des couches sociales et des communautés religieuses.

Bien que les sources soit incertaines et contradictoires, il semble qu'aujourd'hui les trois quarts des membres de la diaspora libanaise se concentrent dans les deux Amériques, dont $30 \%$ aux États-Unis, 15 \% en Argentine, $10 \%$ au Brésil. Le reste est réparti entre l'Afrique, l'Australie, l'Europe et les pays du Golfe.

\section{Fig. 1 : Les Libanais dans le Monde contemporain.}

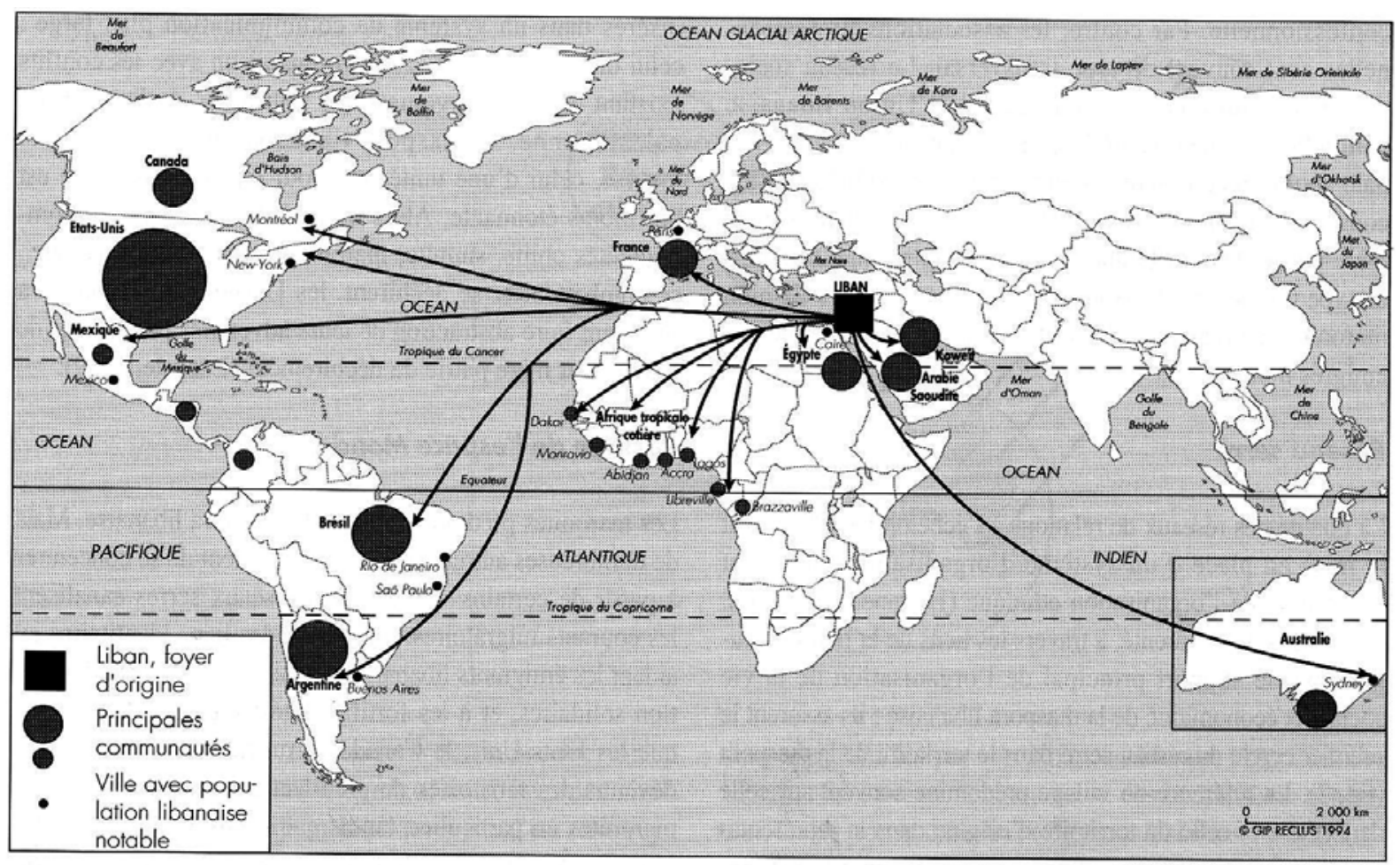

D'après : Chaliand G., Rageau J.-P. (1991) Atlas des diasporas, et l'auteur.

\section{Centre et périphéries}

La logique de constitution d'une diaspora repose sur l'existence d'un centre externe, le territoire de référence, et de périphéries, les pôles de la diaspora. Certaines diasporas ont plus d'un centre : pour les Arméniens l'Arménie historique est le centre principal, mais mythique, tandis que l'Arménie soviétique est le centre secondaire. Pour la diaspora juive, Israël, centre mythique avant 1948, n'est aujourd'hui qu'un centre symbolique, les États-Unis étant le véritable centre. La définition du centre et des périphéries varie en fonction de la conception, du point de repère ou de l'analyse. Pour les Libanais, le territoire du Liban est un centre unique.

D'un côté s'établissent des réseaux ethniques. Les uns engagent le pays d'origine, la diaspora et les pays d'accueil ; d'autres se tissent entre les différents pôles de la diaspora ; ces relations migratoires, financières, commerciales, culturelles, voire politiques, s'appuient sur des réseaux de solidarité familiale et communautaire ; la solidarité se nourrit de contacts constants entre 
les éléments dynamiques de la diaspora, et passe par des actions organisées en vue de maintenir des échanges culturels, économiques et politiques avec le pays d'origine (Sheffer,1986). D'autre part, la diaspora libanaise organise l'encadrement socioculturel et confessionnel dans les territoires d'accueil.

Cette pratique de l'organisation sociale en réseaux semble avoir prédisposé à l'exercice de formes de communication multiples intra- et transnationales. Les technologies modernes de communication apparaissent comme une chance historique nouvelle de survie des diasporas (Raulin, 1991). Les Libanais de la diaspora ont transposé leur tradition journalistique dans les pays d'accueil. Toutes sortes de publications, en langue maternelle et locale, ont été lancées en Égypte, aux États-Unis, dans les pays d'Amérique latine, en Australie et en Europe, en France en particulier. Le développement de la presse diasporique sert non seulement comme moyen d'information au service de la conscience collective et du maintien de l'identité ethnique, mais aussi comme support publicitaire pour les activités économiques de la diaspora et du pays d'origine. La presse renforce ainsi les liens entre le centre et les périphéries.

Les Libanais ont fondé aussi, en raison de leur diversité confessionnelle, leurs propres lieux de culte dans différents pays de la diaspora. Aux États-Unis, à titre d'exemple, on trouve à Dearborn (Michigan) une mosquée pour les chiites (husseinya) et une autre pour les sunnites, une église pour les maronites et une autre pour les grecs, etc. Il existe aussi un grand nombre d'associations socioculturelles, de clubs, de cercles fondés sur une base ethnique, villageoise et confessionnelle. Par contre, les associations professionnelles sont souvent de type transversal. Le réseau socioculturel inclut aussi les branches de l'Union libanaise culturelle mondiale (ULCM), une organisation transnationale apolitique qui gère les affaires de la diaspora et vise à conserver l'identité libanaise et à maintenir les liens avec la mère patrie. Durant la guerre civile, d'autres organisations de type politico-confessionnel ont été créées, telles l'Union maronite mondiale (UMM) et la Ligue islamique chiite des émigrés libanais.

\section{Réseau serré}

La qualité des réseaux de relations à l'échelon local facilitent la mise en place d'un système d'organisation traversant l'ensemble de l'organisation ethnique (Hovanessian, 1992). Les usages de la parenté, à travers les liens de la famille élargie, sont le support principal de l'organisation de la vie sociale et économique de la diaspora libanaise ; ils assurent le premier cercle du réseau serré dans le territoire de la diaspora (fig. 2). La référence au village prédomine souvent sur celle de l'ethnie et celle du territoire d'origine dans la représentation des sujets, surtout des première et deuxième générations. Son pouvoir s'explique par l'empreinte du passé. L'emploi de la notion de village semble matérialiser l'enracinement collectif et la prise de possession physique d'un territoire nouveau. Ce réseau serré se juxtapose à celui de clans familiaux.

La référence à la religion ou plus encore à la confession est un phénomène particulier dans l'organisation spatiale de la diaspora libanaise. À l'intérieur de la diaspora, issue d'un pays pluriconfessionnel (17 communautés religieuses appartenant aux trois grandes religions), on voit se dessiner des réseaux confessionnels. Les pratiques religieuses spécifiques permettent la reproduction de rapports sociaux au sein de la diaspora.

Progressivement se dessinent aussi d'autres références collectives, beaucoup plus abstraites, et prenant cette fois comme norme non plus la spatialisation des pratiques sociales, mais des 
relations basées sur les intérêts communs des sujets, quelles que soient leur origine géographique et leur appartenance familiale ou confessionnelle. L'efficacité des réseaux serrés est toujours perceptible, mais ils ne suffisent plus à l'organisation interne et externe de la diaspora libanaise. Ils conservent leur efficacité à condition d'être insérés dans un système de communication plus large: celui du réseau ethnique. En comparaison avec les conflits existant entre les organisations politiques de la diaspora palestinienne, la diaspora libanaise présente l'aspect inverse, celui d'une unité qui, pour être relative, n'en est pas moins étonnante. Alors qu'au Liban les diverses communautés chiite, sunnite, maronite, orthodoxe, druze, etc., se combattent et se déchirent, les Libanais de la diaspora semblent faire abstraction de leurs adversités pour tenter de sauver leur mère patrie du déchirement (Lacoste, 1989).

Fig. 2 : Diaspora, réseau ethnique et réseau serré.

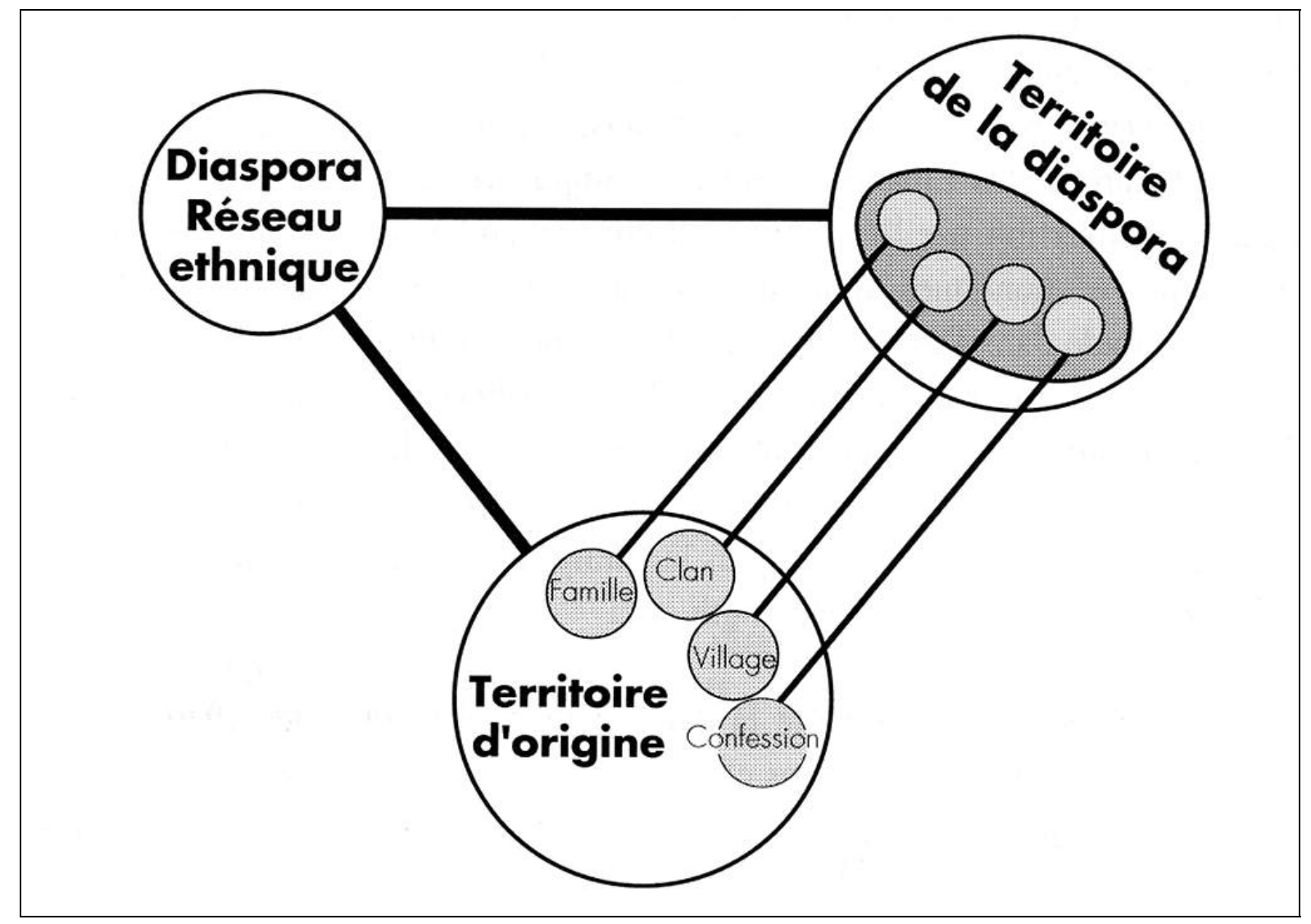

\section{Partage de l'espace Monde}

Les maronites prédominent dans la diaspora libanaise. Mais de nombreuses autres communautés tentent de se concentrer à partir de certains villages. Les réseaux serrés canalisent les courants migratoires, renforçant des liens identitaires en aidant les émigrants libanais à choisir des territoires de fixation solidaires, et à les fortifier ultérieurement. C'est ainsi que les États-Unis, le Canada, l'Australie et l'Europe sont devenus des territoires de prédilection pour les chrétiens, maronites en particulier, tandis que l'Afrique l'est pour les chiites. À l'intérieur même du territoire africain, on remarque une canalisation des courants migratoires à partir des villages libanais vers certains pays du continent. Citons, à titre d'exemple, l'arrivée en Côte-d'Ivoire, surtout depuis les années 1960, des chiites libanais originaires de villages du Liban du Sud comme Qana, Zrérie et Jouaya. Une filière migratoire 
de chrétiens s'est établie à partir d'un autre village du Liban du Sud, Bint Jbil, vers l'État de Michigan aux États-Unis. Les syriaques en ont organisé une vers la Suède. En France, l'émigration des chrétiens arméniens du Liban se dirige vers certaines communes d'Île-deFrance, Issy-les-Moulineaux et Alfortville, ou vers la région Rhône-Alpes et Marseille, en raison de la territorialisation de ces espaces par leurs coreligionnaires arméniens depuis le début du siècle (Hovanessian, 1992). À Montréal, la trajectoire diasporique et la localisation des diasporas libanaise et arménienne sont quasi identiques, en raison de leur proximité de langue et de rite (Sanguin, 1993).

\section{Espaces nouveaux et insertion}

L'exterritorialité de la diaspora libanaise, à l'instar de la diaspora chinoise, trouve son expression dans le type d'activités exercées par les Libanais. Ils sont fortement représentés dans des professions d'interface entre production et consommation : commerçants, négociants, intermédiaires. Ce sont là des activités qui n'impliquent pas d'enracinement territorial mais au contraire nécessitent de la fluidité, du mouvement et des migrations. C'est d'ailleurs cette surreprésentation dans les activités intermédiaires qui permet de les rapprocher de l'archétype de la diaspora, la diaspora juive, et pour les mêmes raisons de circonstance et d'apprentissage (Ma Mung, Abdulkarim, 1991).

Durant des siècles, il a existé à partir du Liban une migration de commerçants, mais elle était le fait d'une élite économique d'origine urbaine. Le modèle de l'ascension sociale des Libanais de la diaspora est exemplaire. Elle repose sur la qualité de ses membres et leur capacité extraordinaire d'adaptation dans leurs nouveaux territoires, à la souplesse dans leurs relations avec les autochtones, à leur connaissance des langues locales. L'insertion des Libanais dans les pays de la diaspora a été réalisée dans des conditions difficiles. Au départ, il s'agit du colportage dans les villages isolés d'Amérique et d'Afrique. De là, on est passé à la boutique de fortune et, pour certains, du petit commerce à la distribution intermédiaire, puis aux grandes affaires commerciales et industrielles. La mobilisation des ressources communautaires et l'utilisation de réseaux de solidarités familiales, villageoises et confessionnelles ont tenu une place prépondérante dans la mobilité sociale des Libanais de la diaspora. Dans les pays du Nouveau Monde, l'insertion des Libanais fut extrêmement rapide et, dès la deuxième génération, on les trouve dans les professions libérales, les administrations et à des postes de responsabilité politique : députés ou sénateurs, gouverneurs et même chefs d'États. Ils sont de grands hommes d'affaires, des industriels, des intellectuels. En particulier, la diaspora libanaise a contribué à l'essor économique du Brésil et de l'Argentine.

La constitution de la diaspora libanaise en Afrique occidentale est due au mouvement d'émigration de commerçants et d'intermédiaires sollicité par la France au début du siècle, afin de renforcer son expansion économique dans cette partie du continent. À partir de cette migration de travail, les marchands libanais ont développé leurs propres économies ethniques et ont préservé leur identité culturelle. On trouve ainsi aujourd'hui en Afrique de puissants hommes d'affaires libanais, qui sont souvent à la tête de véritables monopoles commerciaux et industriels. La diaspora libanaise régit une grande partie de l'économie de certains pays d'Afrique occidentale, notamment la Côte-d'Ivoire et le Sénégal. En Sierra Leone, la diaspora libanaise assure la quasi-totalité du commerce du pays, dont le trafic d'or et de diamant.

En Australie, le commerce est la principale activité de la diaspora libanaise, sous forme de mixed business. Mais récemment certains se sont orientés vers l'industrie et l'agriculture. 
Grâce à leurs diplômes, les nouvelles générations recherchent les professions libérales ou la fonction publique. Dans les nouveaux pôles de la diaspora libanaise comme l'Europe, les Libanais n'ont pas rompu avec leur tradition d'insertion par l'économie. Notre recherche sur les activités économiques de la diaspora de France et son insertion rapide dans l'espace français illustre bien ce phénomène à l'échelle européenne (Abdulkarim, 1991). On observe comment la diaspora libanaise a su utiliser les grandes capitales de l'Europe, Paris et Londres en particulier, comme des espaces relais après la paralysie des activités économiques à partir de Beyrouth.

Le rayonnement international de la diaspora libanaise, sa capacité financière et les positions clés qu'elle occupe dans la plupart des pays d'accueil sont précieux par l'appui logistique qu'ils fournissent au fonctionnement du réseau commercial libanais dans le Monde. Les relations familiales, villageoises et confessionnelles jouent un rôle capital dans le renforcement de ce réseau. L'exemple de la banque est édifiant: l'internationalisation des activités bancaires libanaises est fortement liée à la réémergence de la diaspora depuis le déclenchement de la guerre civile; ce mouvement se traduit par l'implantation de banques libanaises dans les grandes places financières mondiales, ainsi que dans certains pays minuscules considérés comme paradis fiscaux, afin de continuer à gérer les capitaux et les investissements de la diaspora, estimés à plus de 25 milliards de dollars (Karam, 1992); le réseau bancaire est un support efficace et un fer de lance de la « diaspora d'affaires ».

\section{Diaspora et avenir du territoire d'origine}

Quelle que soit la qualité de son insertion dans les sociétés d'accueil, la diaspora libanaise manifeste toujours sa double allégeance en se souciant de l'avenir politique et économique du pays d'origine. Cela s'est traduit, tout au long de l'histoire contemporaine du Liban, par l'aide apportée par la diaspora à la mère patrie, surtout aux moments les plus difficiles, à tous les niveau $\mathrm{x}$ et sous plusieurs formes.

La diaspora a joué un rôle décisif dans la détermination de l'avenir du Liban : les Libanais de la diaspora menèrent, dès avant la première guerre mondiale, une lutte acharnée contre l'Empire Ottoman ; Le Caire, Alexandrie et Paris furent ses trois centres politiques les plus actifs ; au lendemain de la guerre et de l'effondrement de l'Empire Ottoman, la diaspora libanaise, surtout celle des chrétiens, poussa le gouvernement français à créer dans sa zone d'autorité un "Grand Liban » au profit des chrétiens et au détriment de la Syrie. La diaspora prit ensuite une place effective dans la lutte contre le pays mandataire, la France, pour l'accession du Liban à l'indépendance. Durant la guerre civile, la diaspora libanaise, notamment celle des États-Unis, de la France, de la Grande-Bretagne et de l'Arabie Saoudite, n'a pas cessé de multiplier ses efforts et ses contacts, en jouant de son influence politique et économique sur les autorités politiques de ces pays, afin d'arrêter les affrontements et de trouver une solution durable à la crise. Ces efforts ont abouti au pacte de Taëf, qui a permis de rétablir la paix. Il n'est donc pas étonnant de voir aujourd'hui l'un des artisans du pacte, un membre de la diaspora, l'homme d'affaires saoudo-libanais Rafic El-Hariri, à la tête du gouvernement du Liban pour maintenir la paix et reconstruire le pays.

La diaspora a contribué à l'évolution économique et sociale du pays d'origine. Véritable diaspora d'affaires, elle a investi dans tous les domaines commerciaux et industriels. Les flux financiers en direction du Liban représentaient, pour la seule année 1982, 56 \% du produit brut du pays. Depuis, les flux se sont réorientés vers d'autres places financières 
internationales, en attendant des conditions meilleures. Ces flux financiers ont participé à la croissance économique du Liban; des banques ont été fondées grâce aux capitaux de la diaspora, des pays du Golfe en particulier. Ils ont joué aussi un rôle majeur dans l'accélération du processus d'urbanisation et l'amélioration du pouvoir d'achat de la population restée au pays. On doit enfin souligner l'aide multiforme qu'apportent les différents réseaux religieux ou villageois de la diaspora aux projets sociaux, éducatifs, sanitaires sur le territoire d'origine.

\section{Références}

Abdulkarim, A. (1991) Les Libanais en France: tradition migratoire et activités économiques. Poitiers : Université, Thèse.

Corm, G. (1989) « La diaspora libanaise », Hérodote, n53.

Hourani, A. ; Shehadi, N. (1992) The Lebanese in the World: A Century of Emigration. Londres : Centre for Lebanese Studies.

Hovanessian M. (1992) Le Lien communautaire: trois générations d'Arméniens. Paris : Armand Colin.

Karam, Sh. (1992) «Les conditions de retour », Les Cahiers de l'Orient, n²8.

Lacoste, Y. (1989) « Géopolitique des diasporas », Hérodote, n53.

Ma Mung, E. ; Abdulkarim, A. (1991) «China y Libano, una misma diaspora ? » El País, Jueves 20, dossier spécial : Movimiento y Migración.

Raulin, A. (1991) « Minorités intermédiaires et diasporas », Revue européenne des migrations internationales, vol. $7, \mathrm{n}^{\circ} 1$.

Safa, E. (1960) L'Émigration libanaise. Beyrouth : Université Saint-Joseph.

Sanguin, A.-L. (1993) «Les diasporas dans les grandes métropoles canadiennes : le cas de Montréal », Communication présentée au colloque international Les réseaux des diasporas, Chypre.

Sheffer, G. (1986) Modern Diasporas in International Politics. London ; Sydney : Croom Helm. 